Universidad Nacional de Chimborazo

NOVASINERGIA, 2020, Vol. 3, No. 2, junio-noviembre (80-92)

ISSN: 2631-2654

https://doi.org/10.37135/ns.01.06.07

Artículo de Investigación

\title{
Almacenamiento de Energía en forma de Hidrógeno en Hidruro de Magnesio $\mathrm{MgH}_{2}$ Energy storage in the form of Hydrogen in Magnesium Hydride $\mathrm{MgH}_{2}$

\author{
Luis Contreras $\mathbb{1}^{1 *}$, Diana Coello(1) Luis Escobar( ${ }^{2}$ \\ ${ }^{1}$ Dirección de Investigación y Desarrollo, Facultad de Ingeniería Civil y Mecánica, Universidad Técnica de Ambato, \\ Ecuador, 180207; dc.coello@uta.edu.ec, \\ ${ }^{2}$ Facultad de Ingeniería Civil y Mecánica, Universidad Técnica de Ambato, Ecuador, 180207; leescobar@uta.edu.ec,
} \\ * Correspondencia: If.contreras@uta.edu.ec
}

Recibido 18 octubre 2020; Aceptado 23 noviembre 2020; Publicado 01 diciembre 2020

\begin{abstract}
Resumen: $\quad$ En esta investigación se llevó a cabo el estudio de la síntesis, caracterización estructural y análisis térmico de desorción de hidrógeno $\left(\mathrm{H}_{2}\right)$ del hidruro de magnesio $\left(\mathrm{MgH}_{2}\right)$ para almacenamiento de hidrógeno. Las muestras fueron molidas mecánicamente por períodos de 2, 5 y 10 horas en atmosferas contenidas de argón (Ar) e hidrógeno $\left(\mathrm{H}_{2}\right)$ y posteriormente caracterizadas utilizando: (1) difracción de rayos X (XRD), (2) calorimetría de barrido diferencial (DSC) y (3) análisis termogravimétricos (TGA). Las difracciones de las muestras molidas mecánicamente mostraron que existe una reducción en el tamaño de grano lo cual mejora la difusión del $\mathrm{H}_{2}$ reduciendo las temperaturas de deshidrogenación hasta en $118^{\circ} \mathrm{C}$. La máxima cantidad de $\mathrm{H}_{2}$ que se logró obtener de las muestras fue $6.65 \% \mathrm{p} / \mathrm{p}$.

Palabras clave: Absorción, desorción, hidrógeno, hidruro de magnesio, molienda mecánica.

Abstract: In this research, the synthesis, structural characterization, and thermal analysis of hydrogen $\left(\mathrm{H}_{2}\right)$ desorption of magnesium hydride $\left(\mathrm{MgH}_{2}\right)$ for hydrogen storage was carried out. The samples were mechanically grounded for 2, 5, and 10 hours in atmospheres containing argon $(\mathrm{Ar})$ and hydrogen $\left(\mathrm{H}_{2}\right)$. Subsequently, the samples were characterized by (1) X-ray diffraction (XRD), (2) differential scanning calorimetry (DSC), and (3) thermogravimetric analysis (TGA). Diffractions of mechanically grounded samples showed a reduction in grain size, which improves the diffusion of $\mathrm{H}_{2}$ by reducing dehydrogenation temperatures by up to $118{ }^{\circ} \mathrm{C}$. The maximum amount of $\mathrm{H}_{2}$ obtained from the samples was $6.65 \% \mathrm{w} / \mathrm{w}$.
\end{abstract}

Keywords: $\quad$ Absorption, desorption, hydrogen, magnesium hydride, mechanically milling.

\section{$1 \quad$ Introducción}

El mundo actual se ha basado principalmente en el uso de energía para el desarrollo económico y tecnológico. Aproximadamente, el $80 \%$ de energía consumida se produce mediante la quema de combustibles de origen fósil, lo cual ha influido en el aumento de los niveles de contaminación con gases de efecto invernadero a la atmosfera y consecuentemente, el deterioro del medio ambiente y la implicación que esto tiene en los seres humanos. (Sánchez, Linares, \& Inzunza., 2020; Kühne, Sánchez, Roth, \& Tornel, 2019). Con este panorama en mente, es cada vez más común escuchar sobre tecnologías emergentes que permitan suplir la demanda de energía de una manera limpia, eficiente y económicamente viable. Dentro de estos parámetros, el $\mathrm{H}_{2}$ despunta como una posible solución a esta problemática, especialmente como combustible, ya que el producto de su combustión genera vapor de agua (Jiménez Sáez, 2020)

El $\mathrm{H}_{2}$ es el elemento más ligero en la tabla periódica de los elementos y abundante en la naturaleza, sin embrago no se encuentra en estado libre, lo cual hace necesario que se lo genere utilizando compuestos como el agua e hidrocarburos mediante uso de otras formas de energía (Centro Nacional de Hidrógeno, 2020). Por ende podemos inferir que el $\mathrm{H}_{2}$ como tal no representa una fuente primaria de energía, sino más bien un vector energético que permite el almacenamiento de energía y su posterior uso en aplicaciones estacionarias o móviles (Cabezas, 2014; Cid, 2014). 


\section{Unach}

Si bien la iniciativa para el uso de $\mathrm{H}_{2}$ como combustible con la consigna de reducir las emisiones y dejar la dependencia de los combustibles fósiles ha ido consolidándose durante los últimos 10 años, el principal problema que se antepone a su implementación es el almacenamiento del $\mathrm{H}_{2}$, comúnmente se lo almacena en tanques en forma de gas a alta presión o como líquido en temperaturas bajo 0 C (García-Conde, 2010; Fernández-Bolaño, 2005). Evidentemente la gran cantidad de espacio que un cilindro ocupa, combinado con la necesidad de altas presiones o bajas temperaturas, hacen que sea poco práctico el uso de estos métodos.

Una solución para un almacenamiento eficiente es introducir el $\mathrm{H}_{2}$ dentro de una matriz sólida y luego extraerlo a ciertos valores de presión y temperatura establecidos (Gonzáles, 2018). Esta es una propiedad característica de los hidruros, en especial los hidruros de magnesio. Existe gran cantidad de información científica en torno a las propiedades y optimización de la termodinámica y cinética de estos compuestos para su aplicación como almacenadores eficientes de $\mathrm{H}_{2}$ (El Kharbachi et al., 2020; Bellosta et al., 2019; Fernández-Bolaño, 2005).

El magnesio y sus aleaciones son considerados materiales con un gran futuro en el campo del almacenamiento de energía en forma de $\mathrm{H}_{2}$, varias aplicaciones son estudiadas actualmente, entre ellas podemos mencionar a las baterías y aplicaciones móviles a través de celdas de combustible como las más importantes. La razón principal detrás del interés en este material en particular, se basa en la ligereza, alta densidad de energía y rentabilidad económica del mismo (Dornheim et al., 2007; Burstow, 2002).

En el caso puntual de las baterías, el uso del Mg como ánodo ha presentado avances importantes, debido a que potencialmente es una alternativa más económica y amigable con el medio ambiente a las actuales baterías de litio-ion (Li-ion) (Tuerxun, 2015). Sin embargo, existen ciertos parámetros como la densidad energética y los ciclos de carga y descarga (reversibilidad) de las baterías en base de $\mathrm{Mg}$ que deben ser perfeccionados para que este material sea considerado como una alternativa viable a las baterías de Li-ion (MacLaughlin, 2019).

Por otro lado, en aplicaciones móviles, el $\mathrm{Mg}$ es considerado como un medio de almacenamiento de hidrógeno $\left(\mathrm{H}_{2}\right)$; necesario para el correcto funcionamiento de las celdas de combustible, usándolo como compuesto en forma de hidruro $\left(\mathrm{MgH}_{2}\right)$, debido a que este, teóricamente presenta una alta capacidad de almacenamiento de hidrógeno equivalente a $7.6 \%$ p/p (composición porcentual en peso). No obstante, las temperaturas en procesos de absorción y desorción de $\mathrm{H}_{2}$ en el hidruro son muy elevadas $\left(\sim 300{ }^{\circ} \mathrm{C}\right)$ y la cinética muy lenta para aplicaciones comercialmente viables (Crivello et al., 2016; de Rango, Marty, \& Fruchart., 2016). En la literatura científica se ha demostrado que se puede llegar a obtener una mejora en la cinética, introduciendo una microestructura a nanoescala que permita la difusión del $\mathrm{H}_{2}$; la molienda mecánica es una forma fácil y práctica, no solo de generar una microestructura a nanoescala, sino de permitir que se agreguen catalizadores para acelerar los procesos antes mencionados. Pese a esto, las temperaturas de desorción del $\mathrm{MgH}_{2}$ no sufren grandes variaciones $\left(\sim 226{ }^{\circ} \mathrm{C}\right)$, por cuanto, se presenta la necesidad de encontrar métodos que permitan desestabilizar la estructura del $\mathrm{MgH}_{2}$ y de esta manera conseguir una disminución en las temperaturas de desorción del $\mathrm{H}_{2}$ (Zhang, Yan, \& Qu, 2018; Wang, 2017; Contreras, 2017).

El objetivo de esta investigación es sintetizar muestras de hidruro de magnesio mediante la técnica de molienda mecánica durante 2, 5 y 10 horas (h) en atmósferas controladas de Argón (Ar) e $\mathrm{H}_{2}$ para comprobar si los efectos de la molienda mecánica sobre las muestras pueden mejorar las propiedades cinéticas y termodinámicas del $\mathrm{MgH}_{2}$ para almacenamiento de $\mathrm{H}_{2}$.

Este estudio comprende la revisión de las propiedades estructurales de las muestras preparadas mediante molienda mecánica usando la difracción de rayos-X (XRD) para comparar las diferencias en la composición. A continuación, los mecanismos de absorción/desorción del $\mathrm{H}_{2}$ serán evaluados mediante técnicas de calorimetría de barrido diferencial (DSC) y análisis termogravimétrico (TGA) para analizar la cantidad de $\mathrm{H}_{2}$ producido.

\section{Metodología}

Esta investigación está basada en experimentación realizada en laboratorio, donde se utilizaron equipos especializados para la síntesis y posterior caracterización de las muestras conforme se muestra en la figura 1.

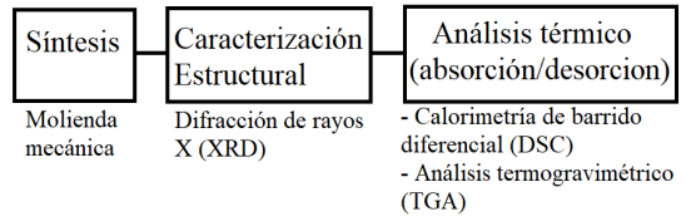

Figura 1: Esquema de las técnicas experimentales usadas en el proyecto. 


\section{Unach}

\subsection{Síntesis}

Como componente inicial, se utilizó polvo de $\mathrm{MgH}_{2}$ (pureza de $95 \%$ fase de hidruro y $5 \% \mathrm{Mg}$; SigmaAldrich Inc.). El polvo de $\mathrm{MgH}_{2}$ fue colocado en un contenedor de acero con bolas de acero de $10 \mathrm{~mm}$ de diámetro para ser molido mecánicamente con una proporción de bolas/polvo de 50:1. La molienda mecánica se llevó a cabo en un molino planetario (Planetary Ball Mill PM 400, Retsch, Birmingham, UK) a $250 \mathrm{rpm}$ por 2, 5 y 10 horas en atmosferas de Ar e $\mathrm{H}_{2}$, con 5 minutos de molienda y 5 minutos de descanso para evitar el sobrecalentamiento de la muestra.

\subsection{Caracterización estructural}

La estructura y morfología de las muestras fue estudiada usando el difractómetro de rayos-X (D8 Advance XRD, Bruker, Birmingham, UK). Los patrones de difracción se analizaron en primer lugar comparándolos con datos de la bibliografía o bases de datos de estructuras cristalinas inorgánicas, por sus siglas en inglés (ICSD) utilizando el software EVA (equipado con la base de datos PDF-2) (DIFFRAC.EVA, Bruker, Version 4.0; 2014). Se realizó un refinamiento por el método de Rietveld con el fin de obtener un análisis cualitativo (determinar la contribución de cada fase en los materiales molidos) utilizando los softwares TOPAS-Academic y jEdit (DIFFRACT.TOPAS, Bruker, Version 4.0; 2014) con datos de referencia obtenidos de la ICSD . Los picos de las difracciones se ajustaron utilizando la función Pseudo-Voight. Además, los parámetros de la red se adaptaron siguiendo los parámetros atómicos (ICSD, Chemical Data Service, Version 2015.1; 2017). Para obtener un ajuste más preciso, todos los puntos anteriores a un valor de $2 \theta$ igual a $25^{\circ}$ se excluyeron del refinamiento.

\subsection{Análisis Térmico}

$\mathrm{El}$ análisis térmico de las muestras de $\mathrm{MgH}_{2}$ comercial y molidas mecánicamente (2, 5 y 10 h) en atmosferas contenidas de $\mathrm{Ar}$ e $\mathrm{H}_{2}$, fue investigado utilizando las técnicas de calorimetría diferencial de barrido. Esta técnica mide la diferencia de flujos de calor entre una muestra y una referencia cuando se calientan o enfrían a una velocidad constante. Esta diferencia de flujo de calor da un pico que corresponde a un evento exotérmico o endotérmico (es decir, cambios de fase, liberación de hidrógeno) que sufre el material estudiado(Höhne, Hemminger, \& Flammersheim, 2003). Para esta investigación se utilizó un equipo calorimetría de barrido diferencial (DSC 204 HP, Netzsch, Birmingham, UK) ubicado dentro de una caja de guantes de atmosfera controlada de Ar, debido a la sensibilidad de las muestras al oxígeno. Alrededor de 10 a $15 \mathrm{mg}$ de las diferentes muestras fueron colocadas en un contenedor de aluminio y analizadas desde temperatura ambiente hasta $\operatorname{los} 500{ }^{\circ} \mathrm{C}$, a una velocidad de calentamiento de $2^{\circ} \mathrm{C} / \mathrm{min}$ y una presión 3 bar en una atmosfera de Ar con un flujo de 100 $\mathrm{ml} / \mathrm{min}$.

Adicionalmente, para investigar la pérdida de masa de las muestras se utilizó la técnica del análisis termogravimétrico, la cual permite medir los cambios de masa del material en función de la temperatura y el tiempo. El equipo consta de una microbalanza, un horno y un controlador, donde las muestras se pueden pesar y calentar/enfriar al mismo tiempo. Las muestras se colocan dentro del horno en un sistema cerrado para controlar la atmósfera. Un gas inerte, en nuestro caso, Ar fluye a través del puerto de gas debajo de la muestra y todos los gases producidos durante el proceso de calentamiento/enfriamiento se llevan al espectrómetro de masas a través de válvulas de escape ubicadas sobre la muestra (TA Instruments, Version 2.1; 2012). En este trabajo se utilizó un equipo de análisis termogravimétricos (TG 209, Netzsch, Birmingham, UK) conectado a un espectrómetro de masas para analizar y registrar los cambios termogravimétricos y la evolución de gas(es) durante el proceso de calentamiento/enfriamiento. Todas las muestras se manipularon y prepararon en una atmósfera inerte de Ar para evitar el contacto con el aire o el agua. Se analizaron muestras de alrededor de $15-20 \mathrm{mg}$ en un contenedor de alúmina con tapa y se calentaron a una velocidad de $2{ }^{\circ} \mathrm{C} / \mathrm{min}$ y una presión de 1 bar de Ar fluyendo a $40 \mathrm{ml} / \mathrm{min}$.

\section{Resultados}

\subsection{Análisis estructural del $\mathrm{MgH}_{2}$ comercial (sin molienda) y molido mecánicamente}

Se analizó la estructura del $\mathrm{MgH}_{2}$ comercial mediante difracción XRD y se determinó que el material consta de $98.3 \% \mathrm{p} / \mathrm{p}$ de $\alpha-\mathrm{MgH}_{2}$ (estructura tetragonal, grupo espacial p42 / mnm 136) (Bortz, Bertheville, Böttger, \& Yvon, 1999) y $1.7 \% \mathrm{p} / \mathrm{p}$ de $\mathrm{Mg}$; el $\mathrm{Mg}$ probablemente se deba a una hidrogenación incompleta durante el proceso de fabricación del $\mathrm{MgH}_{2}$.

La figura 2 ilustra las estructuras del $\mathrm{MgH}_{2}$ comercial y mecánicamente molido durante 2,5 y $10 \mathrm{~h}$ en $\mathrm{Ar}$ obtenido por difracción de rayos X. La difracción del $\mathrm{MgH}_{2}$ molido por $2 \mathrm{~h}$ muestra la formación de una nueva fase consistente con $\gamma-\mathrm{MgH}_{2}$ (ortorrómbica, Pbcn) (Bortz et al., 1999), adicional a las fases correspondientes $\alpha-\mathrm{MgH}_{2}$ y $\mathrm{Mg}$ observadas en el $\mathrm{MgH}_{2}$ comercial. Con el objetivo de cuantificar cada fase presente en la muestra, se llevó a cabo el 


\section{Unach}

refinamiento por el método de Rietveld y se encontró que el $87.4 \% \mathrm{p} / \mathrm{p}$ corresponde a la fase $\alpha-\mathrm{MgH} 2$, el $7.2 \% \mathrm{p} / \mathrm{p}$ a la fase de $\mathrm{Mg}$ y el $5.4 \% \mathrm{p} / \mathrm{p}$ restante a la fase $\gamma-\mathrm{MgH} 2$.

Al moler mecánicamente el $\mathrm{MgH}_{2}$ durante 5 h en Ar, se detectó las mismas difracciones relacionadas con las fases $\alpha$ y $\gamma \mathrm{MgH}_{2}$ y Mg. Sin embargo, la intensidad de los picos de reflexión se redujo y se observó un evidente ensanchamiento de los mismos. El análisis de los parámetros de celda de la muestra molida $5 \mathrm{~h}$ (figura 4) presentan una formación de fases de $85.5 \%$ $\mathrm{p} / \mathrm{p}$ de $\alpha-\mathrm{MgH} 2,8.5 \% \mathrm{p} / \mathrm{p}$ de $\gamma-\mathrm{MgH} 2,2.4 \% \mathrm{p} / \mathrm{p}$ de $\mathrm{Mg}$ y $3.5 \% \mathrm{p} / \mathrm{p}$ de $\mathrm{MgO}$; el $\mathrm{MgO}$ posiblemente se forma debido a la oxidación del $\mathrm{Mg}$ durante la molienda.

Con un mayor tiempo de molienda (10 h) en Ar, se observó que las difracciones de $\mathrm{Mg}, \alpha$ y $\gamma \mathrm{MgH}_{2}$ y $\mathrm{MgO}$ se tornan más amplias y la intensidad decrece, por otra parte, no se detectaron nuevas fases. El refinamiento llevado a cabo (figuras 4 y 5), indicó que la muestra de $\mathrm{MgH}_{2}$ molido por 10 h está compuesta de las siguientes fases cuantificadas de la siguiente manera: $\gamma-\mathrm{MgH}_{2}$ que representa el $38.1 \% \mathrm{p} / \mathrm{p}, \alpha-$ $\mathrm{MgH}_{2}$ el $55.9 \%$ p/p, Mg el $1.6 \%$ p/p) y $\mathrm{MgO}$ el $4.4 \%$ $\mathrm{p} / \mathrm{p}$.

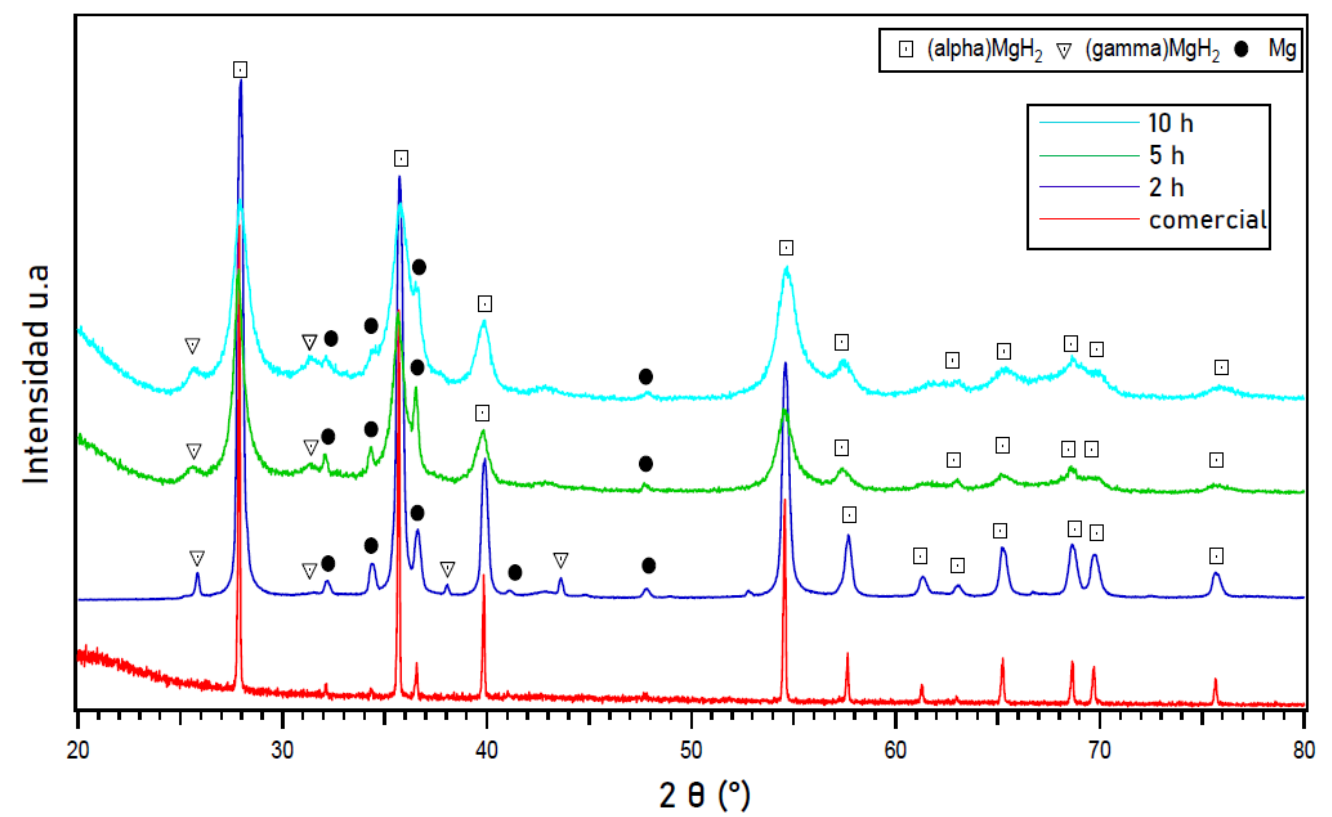

Figura 2: Difracciones de rayos X (XRD) del $\mathrm{MgH}_{2}$ comercial (sin molienda) y molido mecánicamente en una atmósfera contenida de Ar por 2, 5 y 10 horas.

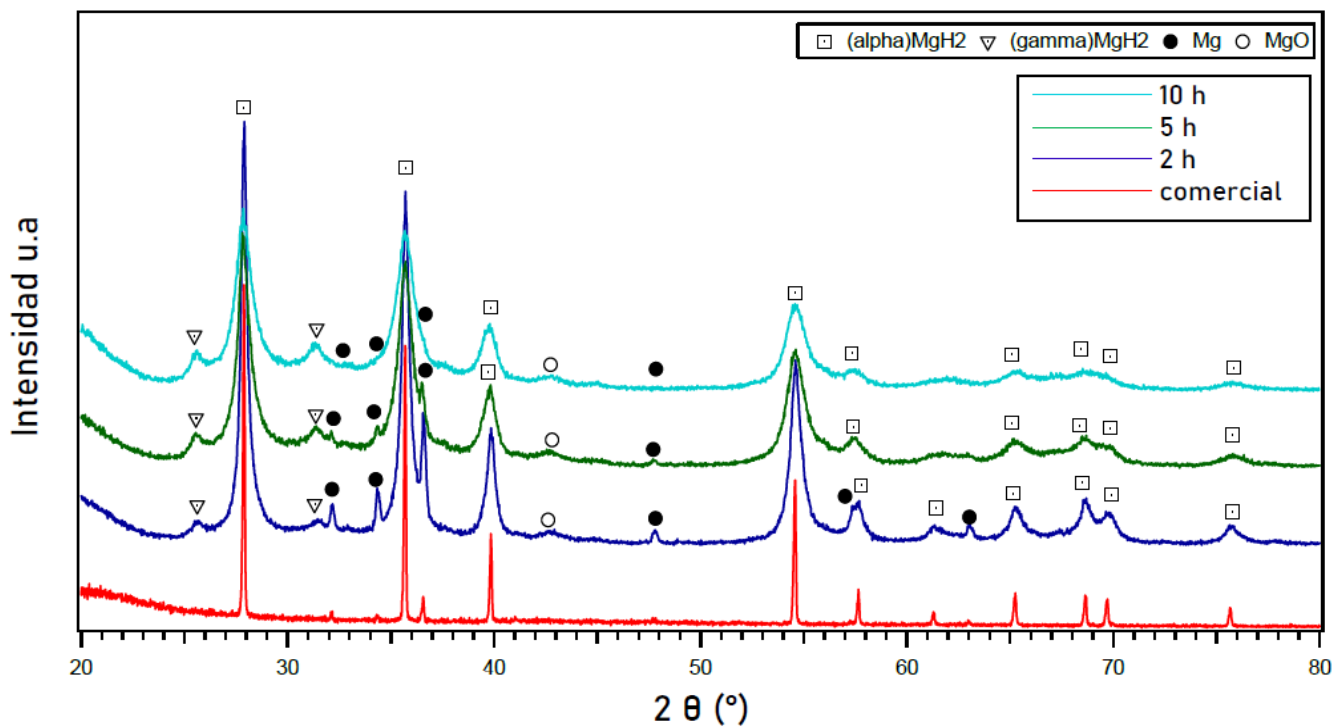

Figura 3: Difracciones de rayos X del $\mathrm{MgH}_{2}$ comercial (sin molienda) y molido mecánicamente en una atmósfera contenida de $\mathrm{H}_{2}$ a presión de 100 bar por 2, 5 y 10 horas. 


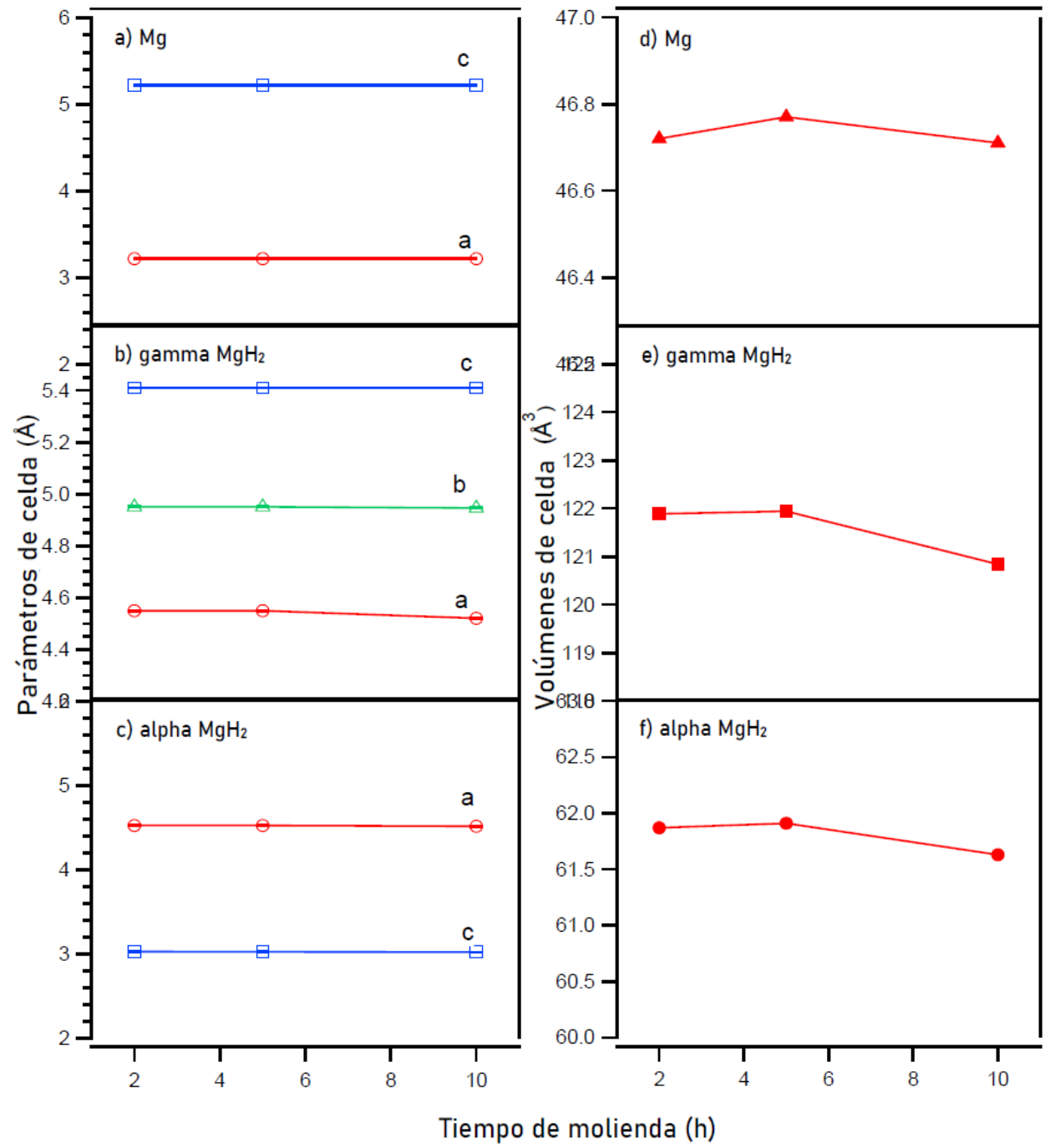

Figura 4: Parámetros de la celda (izquierda) (a) $\mathrm{Mg}$, (b) $\gamma-\mathrm{MgH}_{2}$ y (c) $\alpha-\mathrm{MgH}_{2}$ y volúmenes de celda (derecha) (d) $\mathrm{Mg}$, (e) $\gamma$ $\mathrm{MgH}_{2}$ y (f) $\alpha-\mathrm{MgH}_{2}$ correspondientes al $\mathrm{MgH}_{2}$ molido mecánicamente en atmósfera contenida de Ar por 2, 5 y 10 h en función del tiempo de molienda. Las barras de desviaciones no se observan debido a que son menores que la simbología de los datos.

Por otro lado, se caracterizaron muestras molidas bajo una presión de 100 bar en atmosfera contenida de $\mathrm{H}_{2}$ y sus difracciones se muestran en la figura 3. $\mathrm{El} \mathrm{MgH}_{2}$ molido en $\mathrm{H}_{2}$ durante $2 \mathrm{~h}$ muestra difracciones relacionadas con las fases $\alpha-\mathrm{MgH}_{2}, \gamma-\mathrm{MgH}_{2}, \mathrm{Mg}$ y $\mathrm{MgO}$. En comparación con el material comercial, la intensidad de los picos se reduce y se observa un ensanchamiento en los mismos. El refinamiento realizado sobre la muestra indica los valores de cada una de las fases presentes en el material molido: 79.4 $\%$ p/p de $\alpha-\mathrm{MgH} 2,13.1 \%$ p/p de $\gamma-\mathrm{MgH} 2,2.2 \% \mathrm{p} / \mathrm{p}$ de $\mathrm{Mg}$ y el resto corresponde a $\mathrm{MgO}$.

La muestra molida durante $5 \mathrm{~h}$ en una presión de 100 bar en atmósfera contenida de $\mathrm{H}_{2}$ presenta intensidades reducidas para los picos de $\alpha-\mathrm{MgH} 2$ y
$\mathrm{Mg}$, mientras que las intensidades de $\gamma-\mathrm{MgH} 2$ y $\mathrm{MgO}$ se vuelven más intensas. El refinamiento de la muestra indica que el material está compuesto por $71.2 \% \mathrm{p} / \mathrm{p}$ de $\alpha-\mathrm{MgH} 2,23.04 \% \mathrm{p} / \mathrm{p}$ de $\gamma-\mathrm{MgH} 2,0.6 \% \mathrm{p} / \mathrm{p}$ de $\mathrm{Mg}$ y el $5.2 \% \mathrm{p} / \mathrm{p}$ restante es $\mathrm{MgO}$.

Al moler el polvo de $\mathrm{MgH}_{2}$ durante $10 \mathrm{~h}$ bajo una presión de100 bar en una atmosfera contenida de $\mathrm{H}_{2}$, las difracciones de $\gamma-\mathrm{MgH}_{2}$ y $\mathrm{MgO}$ muestran una mayor intensidad, mientras que las intensidades de los picos de $\alpha-\mathrm{MgH} 2$ y $\mathrm{Mg}$ disminuyen aún más, mostrando un ensanchamiento en los picos. No se detectaron otras fases presentes en la muestra. El refinamiento de la muestra molida por $10 \mathrm{~h}$ permite la cuantificación de fases presentes en la muestra. En este caso, $\alpha-\mathrm{MgH}_{2}$ representa $76.4 \% \mathrm{p} / \mathrm{p}, \gamma-\mathrm{MgH}_{2}$ 


\section{Unach}

muestra $16 \%$ p/p, Mg $2.5 \%$ p/p y el $5.1 \%$ p/p corresponde a $\mathrm{MgO}$.

Los parámetros de celda de las muestras tanto comercial como molidas mecánicamente se encuentran en $\mathrm{Ar}$ e $\mathrm{H}_{2}$ se encuentran resumidas en la tabla 1. Con la finalidad de facilitar la interpretación de los resultados obtenidos en cuanto a los parámetros

Tabla 1: Resumen de los parámetros de celda de las muestras de $\mathrm{MgH}_{2}$ comercial (sin molienda) y molido mecánicamente en atmosferas contenidas de $\mathrm{Ar}$ e $\mathrm{H}_{2}$ por 2, 5 y 10 horas.

\begin{tabular}{|c|c|c|c|c|c|c|c|c|c|c|c|}
\hline & \multirow{3}{*}{$\begin{array}{c}\text { Tiempo } \\
\text { de } \\
\text { molienda }\end{array}$} & \multicolumn{3}{|l|}{$\alpha-\mathrm{MgH}_{2}$} & \multicolumn{4}{|l|}{$\gamma-\mathrm{MgH}_{2}$} & \multicolumn{3}{|l|}{$\mathrm{Mg}$} \\
\hline & & \multicolumn{2}{|c|}{$\begin{array}{l}\text { Parámetros de } \\
\text { celda }(\AA)\end{array}$} & \multirow{2}{*}{$\begin{array}{l}\text { Volumen } \\
\text { de celda } \\
\left(\AA^{3}\right)\end{array}$} & \multicolumn{3}{|c|}{ Parámetros de celda $(\AA)$} & \multirow{2}{*}{$\begin{array}{l}\text { Volumen } \\
\text { de celda } \\
\left(\AA^{3}\right)\end{array}$} & \multicolumn{2}{|c|}{$\begin{array}{l}\text { Parámetros de } \\
\text { celda }(\AA)\end{array}$} & \multirow{2}{*}{$\begin{array}{l}\text { Volumen } \\
\text { de celda } \\
\left(\AA^{3}\right)\end{array}$} \\
\hline & & $\mathrm{a}$ & $\mathrm{c}$ & & $\mathrm{a}$ & b & $\mathrm{c}$ & & $\mathrm{a}$ & $\mathrm{c}$ & \\
\hline \multirow{3}{*}{$\mathrm{Ar}$} & 2 & $\begin{array}{l}4.523 \pm \\
0.002\end{array}$ & $\begin{array}{l}3.025 \pm \\
0.001\end{array}$ & $\begin{array}{l}61.87 \pm \\
0.05\end{array}$ & $\begin{array}{l}4.545 \pm \\
0.007\end{array}$ & $\begin{array}{l}5.413 \pm \\
0.009\end{array}$ & $\begin{array}{l}4.953 \pm \\
0.008\end{array}$ & $\begin{array}{l}121.89 \pm \\
0.03\end{array}$ & $\begin{array}{l}3.215 \pm \\
0.001\end{array}$ & $\begin{array}{l}5.220 \pm \\
0.002\end{array}$ & $\begin{array}{l}46.72 \pm \\
0.04\end{array}$ \\
\hline & 5 & $\begin{array}{l}4.524 \pm \\
0.001\end{array}$ & $\begin{array}{l}3.024 \pm \\
0.001\end{array}$ & $\begin{array}{l}61.91 \pm \\
0.02\end{array}$ & $\begin{array}{l}4.546 \pm \\
0.007\end{array}$ & $\begin{array}{l}5.414 \pm \\
0.009\end{array}$ & $\begin{array}{l}4.954 \pm \\
0.008\end{array}$ & $\begin{array}{l}121.94 \pm \\
0.03\end{array}$ & $\begin{array}{l}3.216 \pm \\
0.001\end{array}$ & $\begin{array}{l}5.220 \pm \\
0.001\end{array}$ & $\begin{array}{l}46.77 \pm \\
0.02\end{array}$ \\
\hline & 10 & $\begin{array}{l}4.516 \pm \\
0.001\end{array}$ & $\begin{array}{l}3.021 \pm \\
0.001\end{array}$ & $\begin{array}{l}61.63 \pm \\
0.02\end{array}$ & $\begin{array}{l}4.519 \pm \\
0.012 \\
\end{array}$ & $\begin{array}{l}5.405 \pm \\
0.012 \\
\end{array}$ & $\begin{array}{l}4.946 \pm \\
0.011\end{array}$ & $\begin{array}{l}120.84 \pm \\
0.06\end{array}$ & $\begin{array}{l}3.216 \pm \\
0.001\end{array}$ & $\begin{array}{l}5.216 \pm \\
0.003 \\
\end{array}$ & $\begin{array}{l}46.71 \pm \\
0.03\end{array}$ \\
\hline \multirow[t]{3}{*}{$\mathrm{H}_{2}$} & 2 & $\begin{array}{l}4.520 \pm \\
0.001\end{array}$ & $\begin{array}{l}3.023 \pm \\
0.001\end{array}$ & $\begin{array}{l}61.77 \pm \\
0.01\end{array}$ & $\begin{array}{l}4.528 \pm \\
0.010\end{array}$ & $\begin{array}{l}5.407 \pm \\
0.012\end{array}$ & $\begin{array}{l}4.961 \pm \\
0.011\end{array}$ & $\begin{array}{l}121.48 \pm \\
0.04\end{array}$ & $\begin{array}{l}3.212 \pm \\
0.001\end{array}$ & $\begin{array}{l}5.216 \pm \\
0.001\end{array}$ & $\begin{array}{l}46.62 \pm \\
0.01\end{array}$ \\
\hline & 5 & $\begin{array}{l}4.524 \pm \\
0.001\end{array}$ & $\begin{array}{l}3.024 \pm \\
0.001\end{array}$ & $\begin{array}{l}61.90 \pm \\
0.01\end{array}$ & $\begin{array}{l}4.531 \pm \\
0.006\end{array}$ & $\begin{array}{l}5.403 \pm \\
0.007\end{array}$ & $\begin{array}{l}4.966 \pm \\
0.007\end{array}$ & $\begin{array}{l}121.58 \pm \\
0.03\end{array}$ & $\begin{array}{l}3.217 \pm \\
0.001\end{array}$ & $\begin{array}{l}5.221 \pm \\
0.001\end{array}$ & $\begin{array}{l}46.81 \pm \\
0.02\end{array}$ \\
\hline & 10 & $\begin{array}{l}4.528 \pm \\
0.001\end{array}$ & $\begin{array}{l}3.023 \pm \\
0.001\end{array}$ & $\begin{array}{l}61.97 \pm \\
0.02\end{array}$ & $\begin{array}{l}4.546 \pm \\
0.003\end{array}$ & $\begin{array}{l}5.408 \pm \\
0.004\end{array}$ & $\begin{array}{l}4.969 \pm \\
0.005\end{array}$ & $\begin{array}{l}122.16 \pm \\
0.02\end{array}$ & $\begin{array}{l}3.339 \pm \\
0.001\end{array}$ & $\begin{array}{l}5.47 \pm \\
0.02\end{array}$ & $\begin{array}{l}52.86 \pm \\
0.03\end{array}$ \\
\hline \multicolumn{2}{|c|}{$\begin{array}{l}\text { Comercial (sin } \\
\text { molienda) }\end{array}$} & $\begin{array}{l}4.518 \pm \\
0.001\end{array}$ & $\begin{array}{l}3.023 \pm \\
0.001\end{array}$ & $\begin{array}{l}61.72 \pm \\
0.02\end{array}$ & & & & & $\begin{array}{l}3.211 \pm \\
0.001\end{array}$ & $\begin{array}{l}5.209 \pm \\
0.001\end{array}$ & $\begin{array}{l}46.53 \pm \\
0.01\end{array}$ \\
\hline
\end{tabular}

Los parámetros de celda a y c después de moler $\mathrm{MgH}_{2}$ durante $2 \mathrm{~h}$ en Ar muestran un incremento en su tamaño y continúan con esta tendencia hasta las $5 \mathrm{~h}$ de molienda, después de este tiempo (10 h molienda), se observa una contracción en la celda unitaria, esta tendencia es congruente en todas las fases presentes en las muestras molidas en Ar.

Por el contrario, los parámetros y volúmenes de celda en las muestras molidas mecánicamente en $\mathrm{H}_{2}$ a 100 bar de presión, denotan una tendencia creciente incluso después de moler la muestra por $10 \mathrm{~h}$.

Los cambios en los parámetros de celda se pueden atribuir a varios factores, entre ellos la deformación o sustitución debido a la molienda mecánica. En el caso puntual de esta investigación es muy probable que estén relacionados con la reducción del tamaño de los cristales (reducción en el tamaño del grano) conforme y volúmenes de celda, se añadieron gráficos complementarios como en las figuras 4 y 5 . De estos podemos mencionar que $\alpha-\mathrm{MgH}_{2}$ tiene una estructura tetragonal con grupo espacial $\mathrm{P} 42 / \mathrm{mnm}, \mathrm{Mg}$ tiene una estructura hexagonal con grupo espacial $\mathrm{P} 63 / \mathrm{mmc}$ y $\gamma$ $\mathrm{MgH} 2$ muestra una estructura ortorrómbica con grupo espacial Pbcn. 


\section{Unach}

$\mathrm{MgH}_{2}$ comercial (Jain, Lal, \& Jain, 2010), es evidente que la capacidad esperada de $\mathrm{H}_{2}$ se reduce. Este efecto se puede explicar debido a las impurezas del material ya que acorde al análisis realizado en el punto 3.1 de esta investigación, el $\mathrm{MgH}_{2}$ comercial no es totalmente puro, sino que contiene una pequeña fracción de $\mathrm{Mg}$ lo que conduce a una menor capacidad de desorción de hidrógeno, además de otras impurezas que pueden estar asociadas con el manejo del material.

La muestra molida mecánicamente durante $2 \mathrm{~h}$ en atmósfera controlada de $\mathrm{Ar}$ mostró que la deshidrogenación comienza alrededor de los $300{ }^{\circ} \mathrm{C}$ y alcanza un máximo pico a los $350^{\circ} \mathrm{C}$, se observó una única reacción endotérmica, ligada a la liberación de $\mathrm{H}_{2}$ del $\mathrm{MgH}_{2}$. Por otra parte, el análisis TGA mostró una cantidad total de $6.60 \% \mathrm{p} / \mathrm{p}$ de desorción de $\mathrm{H}_{2}$ hasta $\operatorname{los} 400{ }^{\circ} \mathrm{C}$.

En la muestra molida $5 \mathrm{~h}$ en atmosfera controlada de Ar, el análisis DSC presentó una fuerte reacción endotérmica que inició aproximadamente a los 290 ${ }^{\circ} \mathrm{C}$, con un pico máximo a los $332.8^{\circ} \mathrm{C}$, seguida de una reacción endotérmica subsecuente que alcanzó un pico máximo a los $340.5^{\circ} \mathrm{C}$ y se desarrolló hasta $387.3^{\circ} \mathrm{C}$. Del análisis TGA realizado en esta muestra, se evidenció que una cantidad total de $6.52 \% \mathrm{p} / \mathrm{p}$ de $\mathrm{H}_{2}$ liberado. Cabe mencionar que la reacción subsecuente observada en el DSC de esta muestra, podría estar asociado con la descomposición de la fase $\alpha-\mathrm{MgH}_{2}$ dado que esta fase se descompone a temperaturas más altas que la fase $\gamma-\mathrm{MgH}_{2}$ (Varin, Czujko, \& Wronski, 2006).

El material molido durante $10 \mathrm{~h}$ en atmósfera controlada de Ar, mostró una desorción de $\mathrm{H}_{2}$ endotérmica en un solo paso, con una temperatura inicial de $251.8^{\circ} \mathrm{C}$ y un pico máximo a los $322.9{ }^{\circ} \mathrm{C}$.

Una cantidad total de $5.69 \% \mathrm{p} / \mathrm{p}$ de desprendimiento de $\mathrm{H}_{2}$ fue detectado mediante el TGA hasta los 356.9 ${ }^{\circ} \mathrm{C}$ cuando terminó la reacción. Es importante señalar que la fuerte reacción endotérmica observada en el DSC está relacionada con la descomposición del $\gamma$ $\mathrm{MgH}_{2}$ en $\mathrm{Mg}$, liberando $\mathrm{H}_{2}$. Con respecto a las muestras anteriormente mencionadas $(2$ y 5 h) se observa una diferencia notable en las temperaturas de desorción, lo que estaría asociado con la disminución en el tamaño de grano de la muestra molida por $10 \mathrm{~h}$.

En la figura 8 se presenta un resumen de las temperaturas de inicio, pico máximo y final obtenidas experimentalmente del análisis DSC y TGA llevado a cabo en las muestras de $\mathrm{MgH}_{2}$ molidas en Ar. Después de analizar las muestras en atmósfera contenida de Ar, se procedió a revisar las muestras que fueron mecánicamente molidas bajo 100 bar de presión y una atmosfera contenida de $\mathrm{H}_{2}$ a través de las técnicas DSC y TGA. La figura 9a indica las reacciones endotérmicas observadas en las muestras de $\mathrm{MgH}_{2}$ comercial y mecánicamente molido por 2,5 y $10 \mathrm{~h}$. Así mismo, los datos obtenidos de la pérdida de masa y la deshidrogenación vs temperatura obtenidos del análisis TGA, son ilustrados en la figura 9b. La disminución en la temperatura de las diferentes muestras puede ser atribuida a la disminución del tamaño de grano conforme al tiempo de molienda.

Después de analizar las muestras en atmósfera contenida de Ar, se procedió a revisar las muestras que fueron mecánicamente molidas bajo 100 bar de presión y una atmosfera contenida de $\mathrm{H}_{2}$ a través de las técnicas DSC y TGA. La figura 9a indica las reacciones endotérmicas observadas en las muestras de $\mathrm{MgH}_{2}$ comercial y mecánicamente molido por 2, 5 y 10 h. Así mismo, los datos obtenidos de la pérdida de masa y la deshidrogenación vs temperatura obtenidos del análisis TGA, son ilustrados en la figura 9b. La disminución en la temperatura de las diferentes muestras puede ser atribuida a la disminución del tamaño de grano conforme al tiempo de molienda.

La muestra molida por $2 \mathrm{~h}$ en $\mathrm{H}_{2}$, presentó una sola reacción de deshidrogenación. El pico relacionado con la desorción de $\mathrm{H}_{2}$ mostrado por el DSC empezó a liberar el gas a una temperatura alrededor de los 278.8 ${ }^{\circ} \mathrm{C}$. La endoterma de esta muestra indica la máxima deflexión a los $340.8{ }^{\circ} \mathrm{C}$. Mediante el análisis TGA se pudo determinar que la muestra libera un total de 6.65 $\% \mathrm{p} / \mathrm{p}$ de $\mathrm{H}_{2}$ hasta los $389.5{ }^{\circ} \mathrm{C}$.

La deshidrogenación del $\mathrm{MgH}_{2}$ molido por 5 h en $\mathrm{H}_{2}$, inició a los $251.8{ }^{\circ} \mathrm{C}$ con un pico máximo a los 334.5 ${ }^{\circ} \mathrm{C}$, seguido de una pequeña reacción hasta los 363.8 ${ }^{\circ} \mathrm{C}$. Por medio del TGA se observó que estas reacciones conjuntas liberan un total de $6.61 \% \mathrm{p} / \mathrm{p}$ de $\mathrm{H}_{2}$ hasta los $387.8{ }^{\circ} \mathrm{C}$. La pequeña reacción subsecuente a la mayor puede atribuirse a la deshidrogenación de la fase $\alpha-\mathrm{MgH} 2$ que ocurre a mayor temperatura que en la fase $\gamma-\mathrm{MgH} 2$

El $\mathrm{MgH}_{2}$ molido durante $10 \mathrm{~h}$ en $\mathrm{H}_{2}$ también muestra una sola reacción endotérmica que comienza a los $249.6^{\circ} \mathrm{C}$ y alcanza un pico máximo a los $319.4{ }^{\circ} \mathrm{C}$, seguido de una pequeña reacción endotérmica a los $371.6^{\circ} \mathrm{C}$. La cantidad total de $\mathrm{H}_{2}$ liberado corresponde a un total de $6.55 \% \mathrm{p} / \mathrm{p}$ según el análisis realizado mediante TGA.

La pequeña reacción subsecuente a la principal en esta muestra está relacionada con la deshidrogenación de la fase $\alpha-\mathrm{MgH} 2$ como se discutió anteriormente.

La figura 10 ilustra las temperaturas de inicio, pico máximo y finales de las muestras de $\mathrm{MgH}_{2}$ molidas bajo una presión de 100 bar en una atmósfera contenida $\mathrm{H}_{2}$ frente al tiempo de molienda (2, 5 y 10 h). 


\section{Unach}

\section{Discusión}

Se investigó el $\mathrm{MgH}_{2}$ comercial y molido mecánicamente durante $2,5,10$ h bajo atmósferas de argón e hidrógeno para comparar el comportamiento del material y los cambios de fase durante el proceso de molienda. En total se sintetizaron y caracterizaron 6 muestras mediante XRD, DSC y TGA.

Las mediciones del XRD después de 2, 5, $10 \mathrm{~h}$ de molienda de $\mathrm{MgH}_{2}$ bajo Ar y 100 bar de $\mathrm{H}_{2}$, mostraron picos correspondientes a la fase $\alpha-\mathrm{MgH} 2$ que fueron menos intensos y más amplios que los del material comercial. Además, se detectaron picos correspondientes a la fase $\gamma-\mathrm{MgH} 2$ en las muestras molidas mecánicamente. El aumento en los anchos de los picos es un indicativo de una reducción en el tamaño de grano, se observó en las muestras experimentalmente sintetizadas, que efectivamente la molienda mecánica contribuye a disminuir el tamaño de grano pasando de $207 \mathrm{~nm}$ para el material comercial (sin molienda) a $18 \mathrm{~nm}$ para el $\mathrm{MgH}_{2}$ molido durante $10 \mathrm{~h}$. Esta reducción en el tamaño de grano contribuye con la disminución de las temperaturas de desorción.

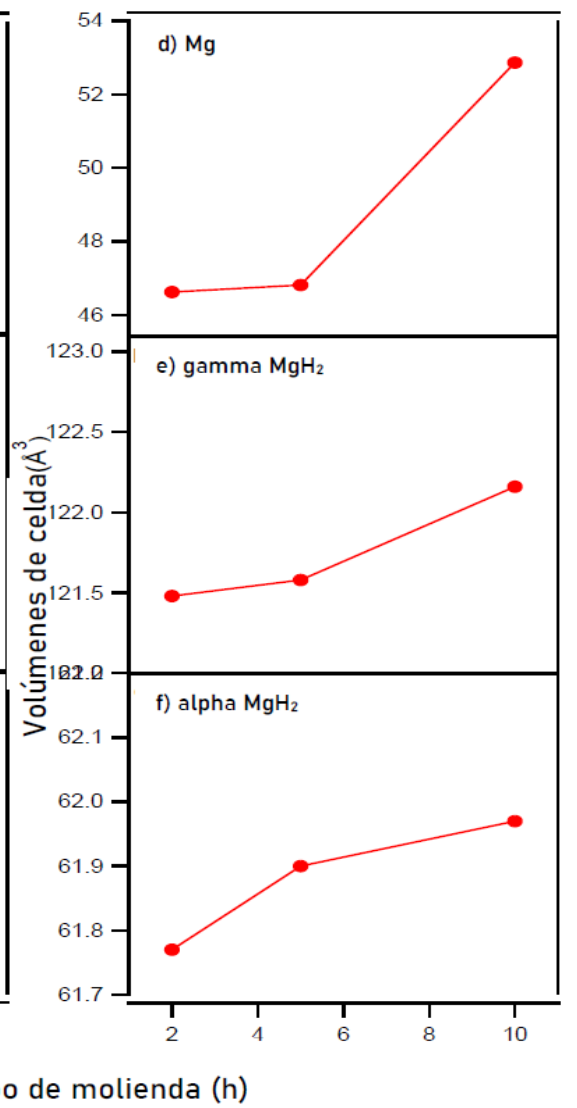

Figura 5: Parámetros de la celda (izquierda) (a) $\mathrm{Mg}$, (b) $\gamma-\mathrm{MgH}_{2}$ y (c) $\alpha-\mathrm{MgH}_{2}$ y volúmenes de celda (derecha) (d) $\mathrm{Mg}$, (e) $\gamma$ $\mathrm{MgH}_{2}$ y (f) $\alpha-\mathrm{MgH}_{2}$ correspondientes al $\mathrm{MgH}_{2}$ molido mecánicamente en atmósfera contenida de $\mathrm{H}_{2}$ a presión de 100 bar por 2, 5 y 10 h en función del tiempo de molienda. Las barras de desviaciones no se observan debido a que son menores que la simbología de los datos.

Los análisis térmicos de los materiales, tanto comerciales como molidos mecánicamente fueron realizados con la finalidad de comprender mejor el comportamiento del material y los mecanismos de descomposición. Las mediciones de DSC demostraron que la desorción de hidrógeno procede en una reacción endotérmica de uno y dos pasos para ambos grupos de muestras molidas mecánicamente en $\mathrm{Ar}$ e $\mathrm{H}_{2}$. Las muestras que presentan reacciones en dos pasos están asociadas con la descomposición de la fase $\gamma-\mathrm{MgH} 2$ que según la literatura se descompone a temperaturas más bajas que la fase $\alpha-\mathrm{MgH} 2$. (Bortz et al., 1999)
En cuanto a las propiedades de almacenamiento de hidrógeno, en las muestras molidas en atmósfera contenida de $\mathrm{Ar}$, la temperatura de inicio de la deshidrogenación se redujo hasta en $118{ }^{\circ} \mathrm{C}$, desde los $370{ }^{\circ} \mathrm{C}$ para el $\mathrm{MgH}_{2}$ comercial (sin molienda mecánica) hasta los $251.8^{\circ} \mathrm{C}$ para la muestra molida durante $10 \mathrm{~h}$. Sin embargo, también se evidenció mediante análisis realizados utilizando el TGA, que la capacidad de almacenamiento de hidrógeno también disminuyó al aumentar el tiempo de molienda, es así que, de los $6.8 \%$ p/p de $\mathrm{H}_{2}$ liberado por el $\mathrm{MgH}_{2}$ comercial, al moler mecánicamente la muestra por 10 


\section{Unach}

h solamente se logró obtener un total de $\mathrm{H}_{2}$ liberado equivalente al $5.7 \% \mathrm{p} / \mathrm{p}$. Que representa $1.1 \% \mathrm{p} / \mathrm{p}$ de $\mathrm{H}_{2}$ menos que la muestra comercial (sin molienda) y corresponde a tan solo el $83.8 \% \mathrm{p} / \mathrm{p}$ de la capacidad total del mismo. Esto se puede atribuir a la contaminación de oxígeno a la que está expuesta la muestra durante los períodos de molienda.

Las temperaturas de desorción de hidrógeno de las muestras molidas bajo presión de 100 bar en una atmosfera contenida de $\mathrm{H}_{2}$, indican una disminución en la temperatura de inicio de la desorción en comparación con las temperaturas del $\mathrm{MgH}_{2}$ comercial de aproximadamente $120{ }^{\circ} \mathrm{C}$, pasando de $370{ }^{\circ} \mathrm{C}$ para el $\mathrm{MgH}_{2}$ comercial (sin molienda) a 249.6
${ }^{\circ} \mathrm{C}$ para el $\mathrm{MgH}_{2}$ molido por $10 \mathrm{~h}$. Esta temperatura es $2{ }^{\circ} \mathrm{C}$ menor que en los resultados obtenidos para las muestras molidas en una atmosfera contenida de Ar.

Nuevamente, en las muestras molidas en $\mathrm{H}_{2}$ analizadas mediante TGA exponen una reducción en la capacidad de hidrógeno de $6.8 \% \mathrm{p} / \mathrm{p}$ para el $\mathrm{MgH}_{2}$ comercial a $6.55 \% \mathrm{p} / \mathrm{p}$ para la muestra molida durante $10 \mathrm{~h}$. Esto representa solo un $0.25 \% \mathrm{p} / \mathrm{p}$ de $\mathrm{H}_{2}$ menos que el obtenido experimentalmente para el $\mathrm{MgH}_{2}$ comercial ( $\sin$ molienda), lo que evidencia que la molienda en $\mathrm{H}_{2}$ tiene un efecto positivo en la deshidrogenación del $\mathrm{MgH}_{2}$ en comparación con las muestras molidas en atmósfera contenida de Ar.

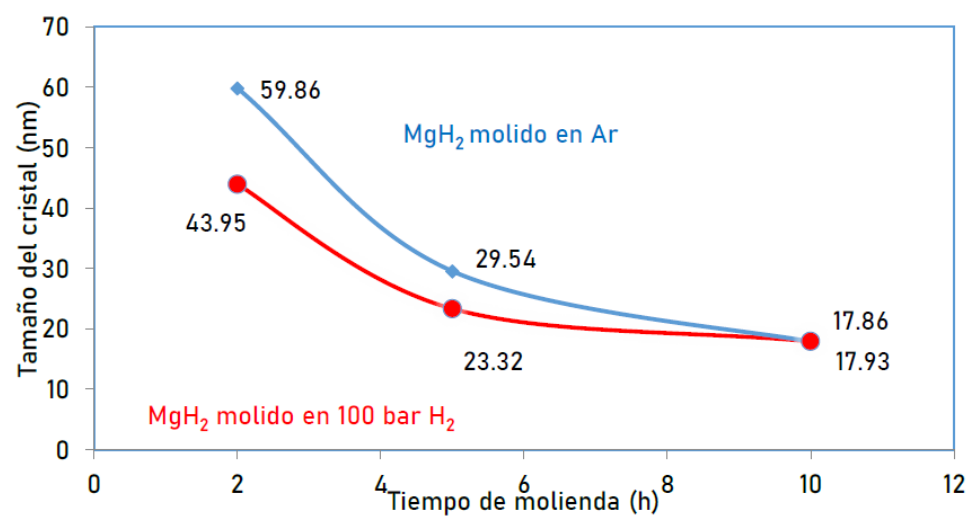

Figura 6: Estimación del tamaño de cristal vs tiempo de molienda mecánica para las muestras de $\mathrm{MgH}_{2}$ molido mecánicamente en atmosferas contenidas de $\mathrm{Ar}$ y 100 bar $\mathrm{H}_{2}$ por 2, 5 y 10 horas.
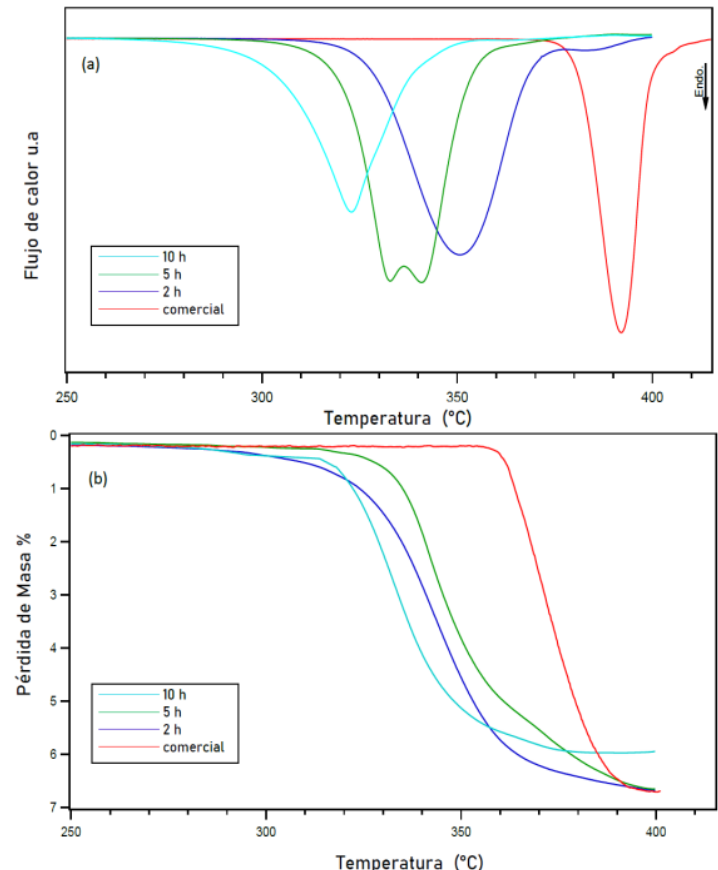

Figura 7: (a) Calorimetría de barrido diferencial. (b) Análisis termogravimétricos de $\mathrm{MgH}_{2}$ comercial (sin molienda) y molido mecánicamente en una atmósfera contenida de Ar por 2, 5 y $10 \mathrm{~h}$. Todos los datos fueron realizados a una velocidad de calentamiento de $2{ }^{\circ} \mathrm{C} / \mathrm{min}$ con un flujo constante de 3 bar de $\mathrm{Ar}$ a $100 \mathrm{ml} / \mathrm{min}$ (DSC) y 1 bar de $\mathrm{Ar}$ con un flujo de $40 \mathrm{ml} / \mathrm{min}$ (TGA). Los datos de DSC y TGA se representan desde los $250{ }^{\circ} \mathrm{C}$ hasta $\operatorname{los} 450{ }^{\circ} \mathrm{C}$ debido a que no se observaron variaciones en rangos anteriores. 


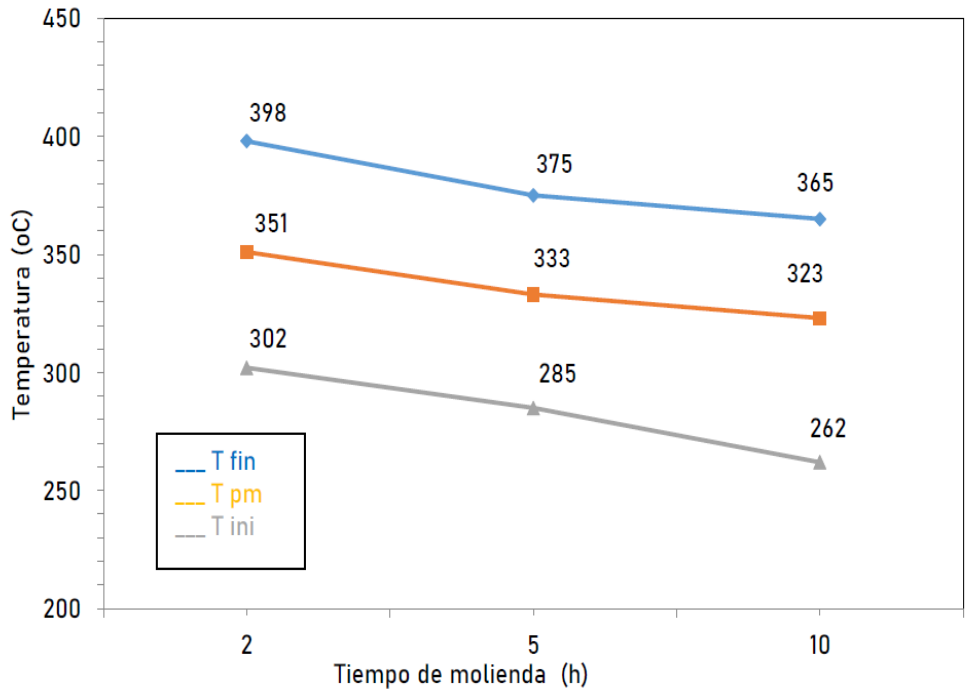

Figura 8: Temperaturas de desorción de $\mathrm{H}_{2}$ (inicial, pico máximo y final) vs tiempo de molienda mecánica para las muestras molidas en atmosfera contenida de Ar.
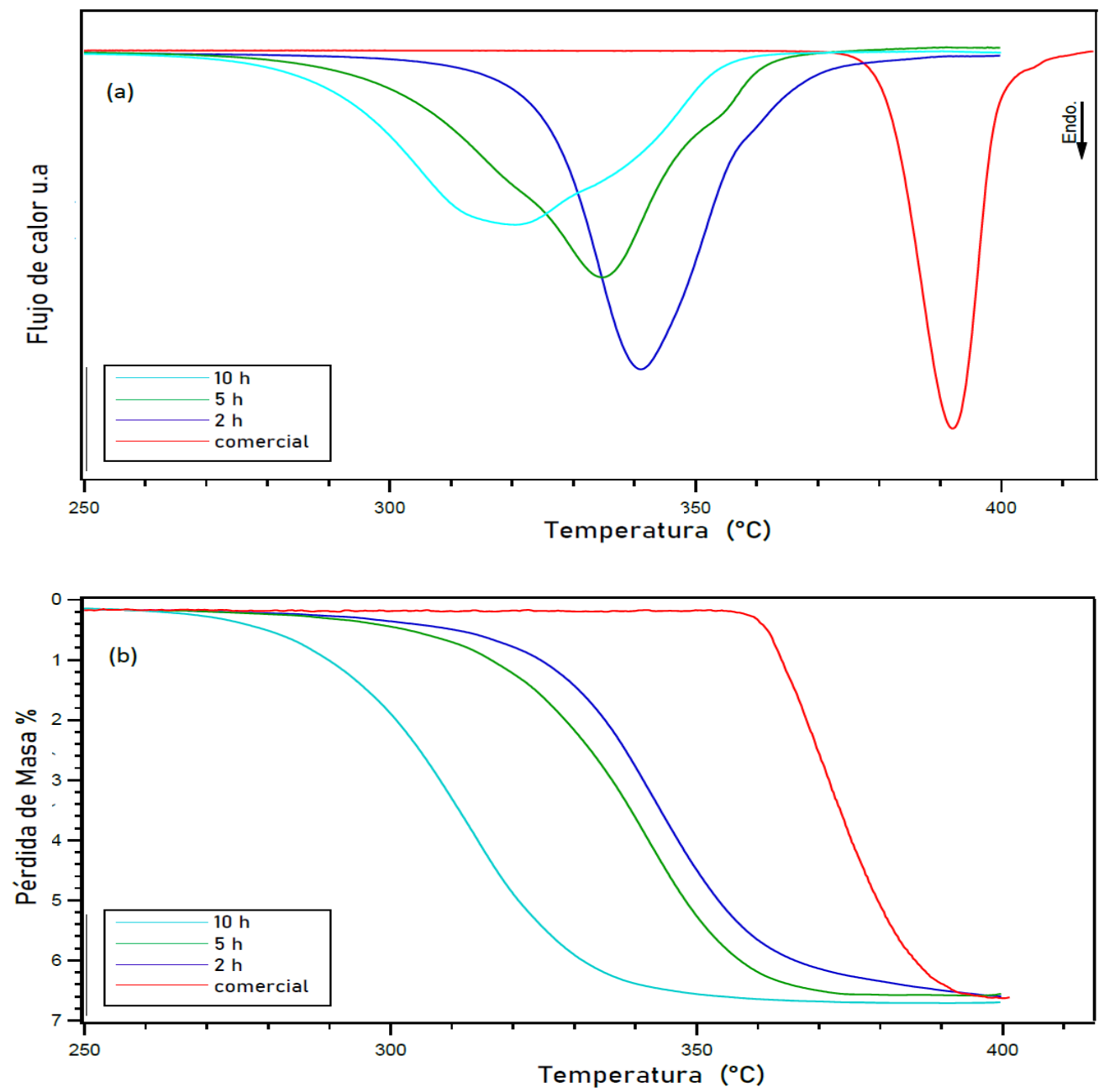

Figura 9: (a) Calorimetría de barrido diferencial. (b) Análisis termogravimétricos de $\mathrm{MgH}_{2}$ comercial (sin molienda) y molido mecánicamente en una atmósfera contenida de $\mathrm{H}_{2}$ a presión de 100 bar por 2, 5 y $10 \mathrm{~h}$. Todos los datos fueron realizados a una velocidad de calentamiento de $2{ }^{\circ} \mathrm{C} / \mathrm{min}$ con un flujo constante de 3 bar de Ar a $100 \mathrm{ml} / \mathrm{min}$ (DSC) y 1 bar de Ar con un flujo 


\section{Unach}

de $40 \mathrm{ml} / \mathrm{min}$ (TGA). Los datos de DSC y TGA se representan desde los $250^{\circ} \mathrm{C}$ hasta los $450{ }^{\circ} \mathrm{C}$ debido a que no se observaron variaciones en rangos anteriores.

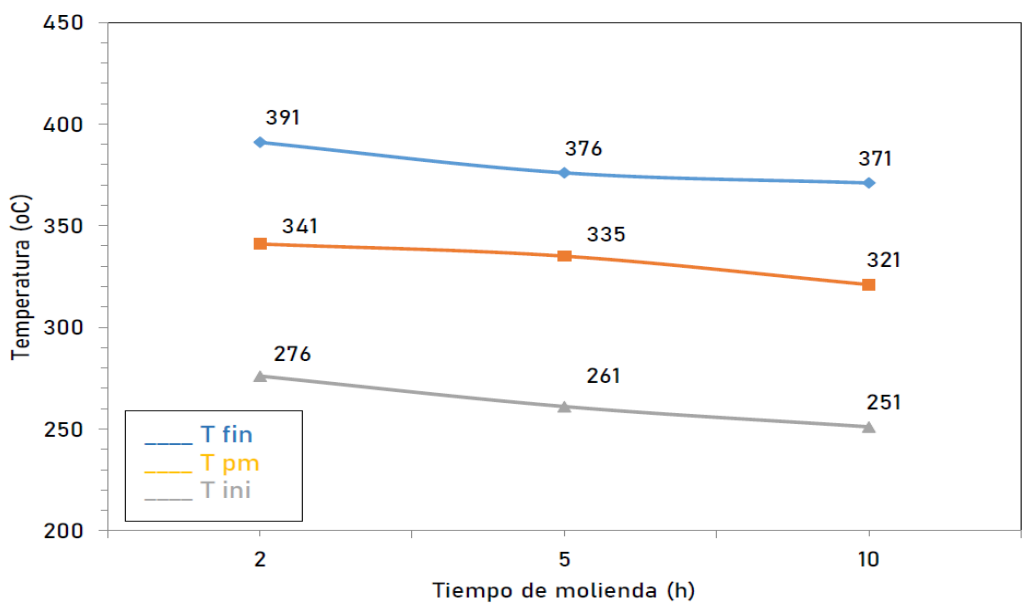

Figura 10: Temperaturas de desorción de $\mathrm{H}_{2}$ (inicial, pico máximo y final) vs tiempo de molienda mecánica para las muestras molidas en atmosfera contenida de $\mathrm{Ar}$.

A partir de los resultados obtenidos en esta investigación, está claro que las propiedades de absorción/desorción de hidrógeno del $\mathrm{MgH}_{2}$ pueden alterarse mediante molienda mecánica. Además, se observó que a medida que aumenta la molienda, la temperatura de desorción de hidrógeno disminuye, pero también lo hace la capacidad de hidrógeno. Sin embargo, cabe mencionar que ninguna de las muestras estudiadas experimentalmente en esta investigación logró acercarse a la capacidad teórica de desorción de $7.6 \% \mathrm{p} / \mathrm{p}$ de $\mathrm{H}_{2}$ declarada en la literatura para el $\mathrm{MgH}_{2}$, esto puede atribuirse a la contaminación de la muestra durante el proceso de molienda.

\section{Conclusiones}

En este trabajo se investigó la síntesis y caracterización de muestras en base a hidruro de magnesio $\mathrm{MgH}_{2}$ para almacenamiento de hidrógeno. Se demostró experimentalmente, que las propiedades termodinámicas de absorción/desorción del $\mathrm{MgH}_{2}$ se pueden mejorar mediante la técnica de molienda mecánica. También se observó que la reducción del tamaño de las partículas mejora la difusión del hidrógeno, reduciendo sustancialmente las temperaturas de deshidrogenación. Las difracciones de rayos $\mathrm{X}$ muestran la aparición de picos relacionados con las fases $\alpha$ y $\gamma-\mathrm{MgH}_{2}$ cuando se muelen mecánicamente las muestras por 2,5 y $10 \mathrm{~h}$. Se pudo también determinar, que el $\mathrm{MgH}_{2}$ molido se descompone en una reacción endotérmica y que la temperatura de deshidrogenación disminuye a medida que aumenta el tiempo de molienda. En cuanto a la capacidad de desorción de hidrógeno, se evidenció por medio de análisis utilizando TGA que mientras mayor sea el período de molienda mecánica, menor va a ser la capacidad de desorción de hidrógeno. Si bien es cierto, estos resultados son alentadores para pensar en el $\mathrm{MgH}_{2}$ como un compuesto que nos permita movernos hacia el uso de hidrógeno comercialmente, existe la necesidad de seguir trabajando para encontrar nuevos aditivos que cumplan con los requisitos termodinámicos de un sistema real y de esta manera llevar a la práctica los resultados de esta investigación.

\section{Conflicto de Interés}

Los autores declaran que no existen conflictos de interés de naturaleza alguna con la presente investigación.

\section{Agradecimiento}

Los autores expresan su agradecimiento al Dr. David Book y la Universidad de Birmingham UK por el soporte y accesibilidad para el uso de los equipos de laboratorio utilizados en esta investigación.

\section{Referencias}

Bellosta, J., Ares, J., Barale, J., Baricco, M., Buckley, C., Capurso, G., Gallandat, N., Grant, D., Guzik, M., Jacob, I., Jensen, E., Jensen, T., Jepsen, J., Klassen, T., Lototskyy, M., Manickam, K., Montone, A., Puszkiel, J., Sartori, S., Dornheim, M. (2019). Application of hydrides in hydrogen storage and compression: Achievements, outlook and perspectives. International Journal of Hydrogen Energy, 44(15), 7780-7808. https://doi.org/10.1016/j.ijhydene.2019.01.104

Bortz, M., Bertheville, B., Böttger, G., \& Yvon, K. (1999). Structure of the high pressure phase $\lambda-\mathrm{MgH} 2$ by 


\section{Unach

neutron powder diffraction. In Journal of Alloys and Compounds (Vol. 287, Issues 1-2, pp. L4-L6). Elsevier Sequoia SA. https://doi.org/10.1016/S09258388(99)00028-6

Burstow, C. (2002). Magnesium: The impact of projected new supply on prices over the next five years. Transactions of the Institutions of Mining and Metallurgy, Section C: Mineral Processing and Extractive Metallurgy, 111(MAY/AUG). https://doi.org/10.1179/mpm.2002.111.2.62

Cabezas, M. (2014). Hydrogen energy vector: demonstration pilot plant with minimal. International Journal of Hydrogen Energy, 39, 18165-18172.

Centro Nacional de Hidrógeno. (2020). Hidrógeno. https://www.cnh2.es/el-hidrogeno/

Cid, I. (2014). Hidrógeno: Vector Energético en el Siglo XXI. https://core.ac.uk/download/pdf/289970908.pdf

Contreras, L. (2017). UBIRA ETheses - Mechanical synthesis of magnesium alloys for hydrogen storage. (PhD Thesis).

https://etheses.bham.ac.uk/id/eprint/8302/

Crivello, J., Denys, R., Dornheim, M., Felderhoff, M., Grant, D., Huot, J., Jensen, T., de Jongh, P., Latroche, M., Walker, G., Webb, C., \& Yartys, V. (2016). Mgbased compounds for hydrogen and energy storage. Applied Physics A: Materials Science and Processing, 122(2), 1-17. https://doi.org/10.1007/s00339-016-9601-1

de Rango, P., Marty, P., \& Fruchart, D. (2016). Hydrogen storage systems based on magnesium hydride: from laboratory tests to fuel cell integration. Applied Physics A: Materials Science and Processing, 122(2), 1-20. https://doi.org/10.1007/s00339-016-9646-1

DIFFRAC.SUITE EVA (Version 4.0) [XRD Software ]. (2014). | Bruker. Retrieved October 18, 2020, from https://www.bruker.com/products/x-ray-diffractionand-elemental-analysis/x-ray-diffraction/xrdsoftware/eva.html

DIFFRAC.SUITE TOPAS (Version 4.0) [XRD Software]. (2014).| Bruker. Retrieved October 18, 2020, from https://www.bruker.com/products/x-ray-diffractionand-elemental-analysis/x-ray-diffraction/xrdsoftware/topas.html

Dornheim, M., Doppiu, S., Barkhordarian, G., Boesenberg, U., Klassen, T., Gutfleisch, O., \& Bormann, R. (2007). Hydrogen storage in magnesium-based hydrides and hydride composites. Scripta Materialia, 56(10), 841-846. https://doi.org/10.1016/j.scriptamat.2007.01.003

El Kharbachi, A., Dematteis, E., Shinzato, K., Stevenson, S., Bannenberg, L., Heere, M., Zlotea, C., Szilágyi, P., Bonnet, J., Grochala, W., Gregory, D., Ichikawa, T., Baricco, M., \& Hauback, B. (2020). Metal Hydrides and Related Materials. Energy Carriers for Novel Hydrogen and Electrochemical Storage. Journal of Physical Chemistry C, 124(14), 7599-7607. https://doi.org/10.1021/acs.jpcc.0c01806
Fernández-Bolaño, C. (2005). Energética del hidrógeno: Contexto, Estado Actual y Perspectivas de Futuro. Universidad de Sevilla. Proyecto de Fin de Carrera. http://bibing.us.es/proyectos/abreproy/3823/fichero/ $0+$ Portada+e+\%C3\%8Dndices.pdf

Gonzáles Delgado, A. (2018). Technological comparison of different hydrogen storage methods. Universidad Politécnica de Valencia. Tesis de pregrado. https://riunet.upv.es/bitstream/handle/10251/144039/ González - Comparación tecnológica entre los diferentes métodos de almacenamiento de hidrógeno.pdf? sequence $=2 \&$ is Allowed $=y$

García-Conde, A. G. (2010). Producción, almacenamiento y distribución de hidrógeno. Recuperado de: http://www2.udg.edu/Portals/88/proc_industrials/5\% 20-\%200tros\%20Combustibles-Hidrogeno.pdf

Höhne, G., Hemminger, W., \& Flammersheim, H. (2003). Differential Scanning Calorimetry. Springer Berlin Heidelberg. $\quad$ https://doi.org/10.1007/978-3-66206710-9

ICSD (Version 2015.1) [Inorganic Chemical Database Service]. (2017) Retrieved October 18, 2020, from http://icsd.cds.rsc.org/search/basic.xhtml;jsessionid= 82761CD648F766CC9CA76BDA84933E21?cdsrdr $=3$

Jain, I., Lal, C., \& Jain, A. (2010). Hydrogen storage in Mg: A most promising material. International Journal of Hydrogen Energy, 35(10), 5133-5144. https://doi.org/10.1016/j.ijhydene.2009.08.088

Jiménez Sáez, F. (2020). Evaluación técnica y económica del uso de hidrógeno verde en aplicaciones para la indL.ustria y desplazamiento de combustible fósil. Universidad de Chile. http://repositorio.uchile.cl/handle/2250/175586

Kühne, K., Sanchez, L., Roth, J., \& Tornel, C. (2019). Más allá de los combustibles fósiles: Transición fiscal en México. https://www.iisd.org/gsi

MacLaughlin, C. (2019). Status and Outlook for Magnesium Battery Technologies: A Conversation with Stan Whittingham and Sarbajit Banerjee. In ACS Energy Letters (Vol. 4, Issue 2, pp. 572-575). American Chemical https://doi.org/10.1021/acsenergylett.9b00214

Sánchez, D., Linares, J., \& Inzunza, K. (2020). Energías limpias: Una necesidad infravalorada. Recuperado de: https://issuu.com/danielasari/docs/maninfo_final

TA Instruments. (Version 2.1) [Análisis termogravimétrico]. (2012). Análisis termogravimétrico - TA Instruments. Recuperado de: https://www.tainstruments.com/productos/thermalanalysis/thermogravimetric-analysis/?lang=es

Tuerxun, F. (2015). A novel rechargeable battery with a magnesium anode, a titanium dioxide cathode, and a magnesium borohydride/tetraglyme electrolyte. http://ir.nsfc.gov.cn/paperDownload/100001354632 $0 . p d f$

Varin, R. Czujko, T., \& Wronski, Z. (2006). Particle size, 


\section{Unach}

grain size and $\gamma-\mathrm{MgH} 2$ effects on the desorption properties of nanocrystalline commercial magnesium hydride processed by controlled mechanical milling. Nanotechnology, 17(15), 3856-3865. https://doi.org/10.1088/0957-4484/17/15/041

Wang, Y., \& Wang, Y. (2017). Recent advances in additiveenhanced magnesium hydride for hydrogen storage. In Progress in Natural Science: Materials International (Vol. 27, Issue 1, pp. 41-49). Elsevier zoinergiol 2020. vol. 3, Num. 2, junio-noviembre

B.V. https://doi.org/10.1016/j.pnsc.2016.12.016

Zhang, J., Yan, S., \& Qu, H. (2018). Recent progress in magnesium hydride modified through catalysis and nanoconfinement. In International Journal of Hydrogen Energy (Vol. 43, Issue 3, pp. 1545-1565). Elsevier

Ltd. 